\title{
Use of near-infrared spectroscopy (NIRS) in cerebral tissue oxygenation monitoring in neonates
}

\author{
Rene Gumulak, Lucia Casnocha Lucanova, Mirko Zibolen
}

\begin{abstract}
Near-infrared spectroscopy (NIRS) is a technology capable of non-invasive, continuous measuring of regional tissue oxygen saturation $\left(\mathrm{StO}_{2}\right)$. StO 2 represents a state of hemodynamic stability, which is influenced by many factors. Extensive research has been done in the field of measuring $\mathrm{StO}_{2}$ of various organs. The current clinical availability of several NIRS-based devices reflects an important development in prevention, detection and correction of discrepancy in oxygen delivery to the brain and vital organs. Managing cerebral ischemia remains a significant issue in the neonatal intensive care units (NICU). Cerebral tissue oxygenation $\left(\mathrm{CStO}_{2}\right)$ and cerebral fractional tissue extraction (cFTOE) are reported in a large number of clinical studies. This review provides a summary of the concept of function, current variability of NIRS-based devices used in neonatology, clinical applications in continuous $\mathrm{CStO}_{2}$ monitoring, limitations, disadvantages, and the potential of current technology.
\end{abstract}

Key words: cerebral monitoring, cerebral tissue oxygenation, near-infrared spectroscopy, neonate

Received: October 10, 2016; Accepted with revision: March 20, 2017; Available online: March 30, 2017

https://doi.org/10.5507/bp.2017.012

Department of Neonatology, Jessenius Medical Faculty in Martin, Comenius University in Bratislava and University Hospital in Martin, Slovak Republic

Corresponding author: Rene Gumulak, e-mail:gumulak2@uniba.sk

\section{INTRODUCTION}

Non-invasive measurement of cerebral tissue oxygenation using near-infrared spectroscopy (NIRS) is attracting increasing attention in neonatology ${ }^{1}$. NIRS provides a continuous assessment of regional cerebral oxygenation $\left(\mathrm{cStO}_{2}\right)$, and cerebral tissue oxygen extraction (cFTOE). Yet, it is not seen as a routine brain monitoring tool in neonatal intensive care units (NICU) (ref., ${ }^{2,3}$ ). The aim of this review is to provide a summary of the relationship between $\mathrm{cStO}_{2}$ and immediate postnatal adaptation, hypoxic-ischemic encephalopathy (HIE), patent ductus arteriosus (PDA), intraventricular hemorrhage (IVH), and respiratory distress syndrome (RDS), and to describe the clinical relevance of this method. Furthermore, the review summarizes the mode of function, current variability of NIRS-based devices used in neonatology, clinical applications of continuous monitoring of cerebral tissue oxygenation. It covers the limitations and disadvantages of current technology and ends with the potential of such technology if implemented in NICU environment.

\section{History of NIRS-based technology}

Transillumination, or the passage of the light through the body, has been studied since the early 1800s ( ref. $\left.^{4}\right)$. Transillumination of the head, first described by Richard Bright in 1831, would be eventually recognized as the first light-based diagnostic technique to identify hydrocephalus ${ }^{4}$. In 1977, Jobsis reported that the tissue absorbs light (wavelength of 700-1,000 nm), and this fact can be used for vital tissue measurement in the diameter of 5-6 cm. He used a model of the myocardium and brain tissue ${ }^{5}$.
In 1985, Ferrari et al. published human cerebral oximetry studies using NIRS (ref. ${ }^{6}$ ). Since the first report of pediatric application of NIRS for monitoring cerebral oxygenation in sick preterm infants in 1986, the possibilities of NIRS monitoring have significantly increased ${ }^{7}$. A major milestone in terms of availability of equipment capable of continuous monitoring was the year 1993. In May 1993, INVOS 3100® (Somanetics Corporation, Troy, Mich., USA) entered the market as the first commercial cerebral oximetry device approved by the Food and Drug Administration (FDA) $\left(\right.$ ref. $\left.^{8}\right)$. The discovery that functional activation of the human cerebral cortex can be explored by NIRS, has added a new dimension to the research. To obtain simultaneous multiple and localized information, a further major step forward was achieved by introducing NIR imaging and tomography ${ }^{9}$.

\section{Physical principles of NIRS}

Visible light (wavelength $450-700 \mathrm{~nm}$ ) is strongly attenuated as a result of powerful absorption and scattering by the tissue constituens ${ }^{10}$. Near Infrared Spectroscopy (NIRS) is a type of vibrational spectroscopy that employs photon energy in the energy range of $2.65 \times 10^{-19}$ to 7.96 x $10^{-20} \mathrm{~J}$ (corresponding the wavelength range of 750 to $2,500 \mathrm{~nm}$ ). This energy range is higher than necessary to promote molecules only to their lowest excited vibrational states (through a fundamental vibrational transition). On the other hand, the energy range is lower than typical values necessary for electron excitation in molecules (except for some rare earth compounds). The overall objective of spectroscopy is to probe a sample in order to acquire qualitative and/or quantitative information coming from 
the interaction of near-infrared electromagnetic waves with its constituents ${ }^{11}$. Near-infrared spectroscopy has been used as a tool to determine the redox state of light absorbing molecules. This technology is based on the Beer-Lambert Law, which states that light transmission through a solution with a dissolved solute decreases exponentially as the concentration of the solute increases ${ }^{8}$. A decrement in transmitted light intensity is equivalent to the quantity of the substance and the amount of light absorbed by a unit quantity of that substance, defined as the extinction coefficient, a factor that varies with the substance and the incident - light wavelenght ${ }^{4,8}$. In mammalian tissue, only three compounds change their spectra when oxygenated: cytochrome aa3, myoglobin, and hemoglobin. Because the absorption spectra of oxyhemoglobin and deoxyhemoglobin differ, their relative concentrations change within tissue with oxygenation, and the relative concentrations of the types of hemoglobin can be determined $^{12}$.

All NIRS devices emit light at wavelengths within the above mentioned spectrum, and analyze photons returning to the transducer. Because the change in the intensity of the reflected light is dependent upon the oxyhemoglobin to deoxyhemoglobin ratio, oxyhemoglobin saturation can be derived ${ }^{13}$. The ratio between oxygenated and deoxygenated hemoglobin is known as a tissue oxygenation index (TOI). The value of the regional oxygenation or oxygen saturation $\left(\mathrm{StO}_{2}\right)$ acquired by a NIRS-based device is a combination of intravascular oxygenated/deoxygenated venous and arterial (capillary) hemoglobin in a ratio estimated by the manufactor ${ }^{8,9}$. Pulse oximetry deliberately considers only the arterial compartment at the time getting the measurements ${ }^{14}$. Fractional oxygen extraction (FOE) is the ratio between oxygen consumption and oxygen delivery. This is calculated using the formula $\mathrm{FOE}=\left(\mathrm{SpO}_{2}-\mathrm{StO}_{2}\right) / \mathrm{SpO}_{2}$, without measuring the flow. Assuming constant cerebral oxygen consumption, the value will rise as oxygen delivery to the brain falls, until maximum oxygen extraction is achieved ${ }^{15,16}$. Unlike pulse oximetry, NIRS is not dependent on pulse wave, and it uses more wavelengths for spectroscopic transillumination, therefore characterizing more chromophores ${ }^{10,14}$. The extremely low level of absorption of NIR light by hemoglobin is the dominant factor in achieving measurement requiring low light intensity. While benchtop co-oximeters utilize multiple wavelengths to differentiate various dyshemoglobins in vitro, noise reduction remains the most important factor in improving accuracy and precision in vivo ${ }^{17}$.

\section{Cerebral tissue oxygenation in Neonatology}

The neonatal period is very unique, as the infant undergoes dramatic physiologic changes during transition from intra to extra uterine life. This process involves changes in hemodynamics and affects oxygenation, while reflected in $\mathrm{StO}_{2}$ values ${ }^{11}$. Measuring cerebral oxygenation using NIRS has taken on an increasing important role in neonatal care. Several companies have developed commercial devices, and more publications are reporting absolute boundary NIRS values or percentiles for neonates ${ }^{1}$. NIRS is sensitive to all tissues penetrated by the light, for example, on the head these represent skin, scalp, skull, sub-arachnoid space, and grey and white brain matter. The skin, the scalp and the skull are much thinner in neonates, and instruments with a multi-distance approach cancel out the influence of the superficial tissue. Furthermore, NIR spectroscopy is less sensitive in larger compared to smaller blood vessels ${ }^{18,19}$.

A large number of studies on NIRS focused on functional brain imaging has been published. Compared to electroencephalography (EEG), NIRS is less susceptible to data corruption by movement artifacts, and offers more highly spatially resolved images of activation. Blasi et al. demonstrated that spatial mapping and size of activation in infants have a high degree of reliability ${ }^{20}$.Several studies using different NIRS devices have described the changes in cerebral regional tissue oxygenation during the first minute of life. Reference ranges and centile charts have already been established for $\mathrm{cStO}_{2}$ and cFTOE during the first 15 min after birth ${ }^{21}$.

\section{Reference ranges}

The current literature indicates correlation between irreversible mitochondrial damage and the total energy disruption that lasts 30 to $120 \mathrm{~min}$ at values ranging from 33 to $45 \%$. The studies were conducted on piglets mod$\mathrm{els}^{22,23}$. Since the premature brain is extremely vulnerable, especially in hemodynamically unstable neonates, monitoring cerebral oxygenation has a high priority and is increasingly combined with other brain monitoring devices such as amplitude-integrated EEG (ref. ${ }^{19}$ ). Lemmers et al. compared simultaneously determined left and right fronto-parietal measurements of $\mathrm{cStO}_{2}$ with INVOS ${ }^{\mathrm{TM}}$ 5100 (Somanetics, Troy, Michigan, USA) and referred

Table 1. NIRS technology evolution ${ }^{4-6,8}$.

\begin{tabular}{ll}
\hline Year & Information \\
\hline 1831 & Translumination of the head, first described by Richard Bright \\
1881 & The first (near) infrared spectra measured by Abney and Festing \\
1905 & W.W. Cobletz published the results of a large study using spectra measurement \\
1950 & The first industrial applications \\
1975 & Chemometrics \\
1977 & Jobsis published the study using the model of the myocard and brain tissue \\
$1985-1990$ & Light-fiber optics \\
1993 & INVOS 3100® (Somanetics Corporation, Troy, Mich., USA) \\
\hline
\end{tabular}


similar values. Values of cerebral oxygen saturation were lower in all regions on day 7 compared to day 1 of life. However, the longitudinal study showed a slight tendency for $\mathrm{cStO}_{2}$ values to increase at the right fronto-parietal position at day 3 . This pattern changed during unstable arterial oxygenation with substantial drops of $\mathrm{SaO}_{2}$ with or without subsequent hyperoxemia, when extra oxygen was added for a quick recovery of arterial saturation ${ }^{24}$. In term infants at birth, $\mathrm{cStO}_{2}$ rapidly adapts to extrauterine life and after the $7^{\text {th }}$ minute remains stable. The results are beneficial in terms of demonstration of cerebral oxygenation, documenting the increase in cerebral blood flow in the first minutes of life ${ }^{25,26}$. Karen et al. used NIRO-300 ${ }^{\mathrm{TM}}$ (Hamamatsu Photonics, Hamamatsu, Japan) and published that newborns delivered by vacuum extraction had significantly higher tissue hemoglobin index (THI) 10 to 15 min after birth. Tissue oxygenation index (TOI) and heart rate (HR) were significantly higher in the first $5 \mathrm{~min}$ and $\mathrm{StO}_{2}$ in the first $10 \mathrm{~min}$, but then they did not differ from those in infants after cesarean section ${ }^{27}$.

The study performed by Kratky et al. demonstrated that $\mathrm{cStO}_{2}$ increased rapidly from minute 2. Cerebral fractional oxygen extraction which was calculated for each minute, showed a significant decrease from minute 2 until min 4 and it increased within the first 14 min after delivery. $\mathrm{cStO}_{2}$ significantly showed no further changes after $5 \mathrm{~min}^{28}$.

Pichler et al. defined reference ranges and percentile charts for $\mathrm{cStO}_{2}$ and cFTOE using INVOS ${ }^{\mathrm{TM}} 5100$ (Somanetics, Troy, Michigan, USA) in a large cohort of term and preterm neonates without any need of medical support during the first $15 \mathrm{~min}$ after birth, and they found no significant differences comparing term and preterm neonates ${ }^{29}$. The normal reference range of $\mathrm{cStO}_{2}$ for preterm infants depends on multiple factors such as instrument design, postnatal age, or current clinical status $^{30}$. Cerebral hemoglobin oxygenation correlates with chronological age, but not with postmenstrual age. $\mathrm{cStO}_{2}$ is not correlated with postmenstrual age, but varies with chronological age and hemoglobin concentration in the blood, suggesting that it depends on systemic changes and does not reflect changes associated with brain development ${ }^{31}$. This is consistent with the findings that $\mathrm{cStO}_{2}$ may correlate with the heart and respiratory rate and with arterial $\mathrm{SO}_{2}$ in newborns ${ }^{32}$. Significantly lower $\mathrm{SpO}_{2}$ and heart rate values have been reported in infants born by cesarean section ${ }^{33}$. In the studies evaluating $\mathrm{cStO}_{2}$ measurements in different brain regions in stable preterm and term neonates in the first week of life, it was shown that limits of agreement were quite large and varied between $\pm 14 \%$ and $\pm 18 \%$ (INVOS ${ }^{\mathrm{TM}} 5100$; Somanetics, Troy, Michigan, USA) (ref. ${ }^{34,35}$ ). These results suggest that single site recording of the regional cerebral oxygen saturation and cerebral fractional tissue oxygen extraction can monitor trends in individual patients to detect changes larger than the limits of agreement, but lacks the precision to be used as a robust quantitative variable of cerebral oxygenation ${ }^{36}$.

McNeill et al. confirmed that continuous, long-term $\mathrm{StO}_{2}$ monitoring of premature infants in the NICU is both safe and feasible. Neonatal daily baseline $\mathrm{StO}_{2}$ values measured by the INVOS ${ }^{\mathrm{TM}} 5100$ (Somanetics, Troy, Michigan, USA) not only change with postmenstrual age, but also decrease in the variability of $\mathrm{StO}_{2}$. The group mean $\mathrm{cStO}_{2}$ fell within the expected range of $60-80 \%$, although several subject demonstrated cerebral values consistently above $80 \%$ during the first week of life before declining ${ }^{37}$.

\section{Cerebral oxygenation and HIE}

Neonatal HIE (hypoxic-ischemic encephalopathy) is a major cause of mortality, morbidity, and long-term neurological deficits ${ }^{38}$. Despite the progress in diagnostic modalities, accurate prediction of outcome in neonates with HIE remains a great challenge. MRI can predict outcome reliably only in severe HIE cases. Early EEG findings (24 h) do not provide additional support for outcome prediction and are nonspecific, possibly in relation to medication use ${ }^{38}$. $\mathrm{cStO}_{2}$ was significantly higher between 24 and 48 weeks of age in neonates with HIE with adverse outcomes as compared to those with favourable outcomes, suggesting a decrease in cerebral oxygen consumption during secondary energy failure. Newborns with proven brain injury caused by $\mathrm{HIE}$ have higher values of $\mathrm{cStO}_{2}$ than newborns without brain injury ${ }^{39}$. Plomgaard et al. investigated the benefits and harms of monitoring cerebral oxygenation by NIRS combined with an evidence-based treatment guideline versus no NIRS data and treatment as usual in the group during the first $72 \mathrm{~h}$ of life. Treatment guided by NIRS reduced the burden of cerebral hypoxia without affecting the selected EEG or blood biomarkers ${ }^{40}$.

\section{Cerebral oxygenation and patent ductus arteriosus (PDA)}

The ductus arteriosus (DA) is an important vascular connection between main pulmonary artery and the aorta. After birth, the DA undergoes active constriction and eventual obliteration. PDA occurs when the ductus fails to close completely after delivery ${ }^{41}$. The incidence of hemodynamically relevant DA has been known to affect perfusion of important organ systems such as the brain. Lemmers et al. measured NIRS values in 20 infants with PDA who underwent treatment with indomethacin (gestational age under 32 weeks). The mean arterial blood pressure and $\mathrm{cStO}_{2}$ were significantly lower, and FTOE was significantly higher before the treatment of PDA compared to the control group. Indomethacin had no additional negative effect on cerebral oxygenation. Hemodynamically significant PDA had a negative effect on cerebral oxygenation in the premature infant ${ }^{42}$. NIRS can be used as a screening tool for PDA, it shows encouraging efficiency in identifying ELBW infants who are likely to benefit from early echocardiography and subsequent intervention to close a PDA ( ref. $^{43}$ ).

Vanderheagen et al. analysed a significant increase in $\mathrm{cStO}_{2}$ with concomitant decrease in FTOE at the time of surgical ligation followed by return to baseline values. As a result, the ductal clipping has no negative effect on the cerebral oxygenation ${ }^{44}$. Ductal ligation poses a risk for a further decrease in already compromised cerebral oxygenation in preterm infants. During surgery, median range of $\mathrm{cStO}_{2}$ dropped very slightly. Eleven infants showed a 
drop in blood pressure and 13 infants a drop in $\mathrm{cStO}_{2}$ during surgery accompanied by a decrease in EEG amplitude $^{45}$.

\section{Cerebral oxygenation and respiratory distress syndrome (RDS)}

During artificial ventilation due to RDS, systemic hemodynamics can be disturbed with consequent compromise of cerebral oxygenation. RDS is strongly related to low and fluctuating arterial blood pressure ${ }^{18}$. Lemmers et al. investigated the relationship between mean arterial blood pressure (MABP), $\mathrm{cStO}_{2}$ and FTOE, during the first $72 \mathrm{~h}$ of life. RDS infants showed more periods of positive correlation between MABP and $\mathrm{cStO}_{2}$ and negative correlation between MABP and FTOE. Although Lemmers et al. found that the patterns of cerebral oxygenation and extraction in RDS infants were not different when compared to infants without RDS. They suggested that the frequent periods with possible lack of cerebral autoregulation in RDS infants may cause these infants to be more vulnerable to cerebral damage ${ }^{46}$. Because cerebral oxygenation is essential, $\mathrm{cStO}_{2}$ monitoring can be an important tool to avoid disturbances between carbon dioxide tensions and impact on complications of this process ${ }^{29}$.

Cerebral oxygenation and peri/intraventricular hemorrhage

Sorensen et al. found that there was a significant negative correlation between the severity of the intraventricular hemorrhage and the cerebral oxygenation ${ }^{47}$. Zhang et al. investigated the relationship between intraventricular hemorrhage (IVH) and $\mathrm{cStO}_{2}$ in preterm infants. They founded higher $\mathrm{cStO}_{2}$ values in the first $3 \mathrm{~h}$ after birth in neonates who later developed IVH ( ref. $^{48}$ ). Higher $\mathrm{cStO}$ and lover cFTOE values within $24 \mathrm{~h}$ before detection of IVH have been reported in another study referring compliant results in very preterm infants ${ }^{49}$. It has been proposed that monitoring of $\mathrm{cStO}_{2}$ can potentially reduce the damage to the vulnerable preterm brain.

\section{CONCLUSION}

NIRS is a fascinating and dynamically evolving technology with over 40 years of history. This non-invasive bedside method is both safe and feasible. It enables continuous, long-term $\mathrm{cStO}_{2}$ monitoring of NICU patients. Currently available NIRS based devices use the above technology principles. They only differ is in the number of absolute values of wavelength, as well as in computational algorithms translating measured changes into light attenuation. The use of various optical probes complicates comparison of the results referred in older NIRS studies with the currently published data.

Because large infant population-based normative data are lacking, further studies are needed in order to establish normative data for newly available commercial NIRS instruments. In the future, it is essential to provide the $\mathrm{StO}_{2}$ measurement by means of NIRS based devices with improved quantitative precision, so that this technology will become consistent and usable for NICU worldwide.
However, NIRS provides a unique insight into the possibilities of diagnosing and treating diseases which are typically initially asymptomatic. This promising technology can be used in combination with EEG and ultrasonography in order to provide coherent information, thus improving non-invasive monitoring.

\section{Search strategy and selection criteria}

Scientific databases (PubMed and/or Web of Science) were searched for articles describing methods and applications of NIRS in continuous monitoring in neonates. Articles were searched from 1999 to April 2016 and were selected according to their revelance to clinical use, results and devices used. Articles describing the historical evolution and physical principles of NIRS, which were published earlier, were also included. Only English language papers were reviewed.

\section{ABBREVIATIONS}

cFTOE, Cerebral fractional tissue oxygen extraction; $\mathrm{cStO}_{2}$, Regional cerebral oxygen saturation; DA, Ductus arteriosus; EEG, Electroencephalography; ELBW, Extremely low birth weight; FDA, Food and drug administration; FTOE, Fractional tissue oxygen extraction; HIE, Hypoxic-ischemic encephalopathy; IVH, Intraventricular hemorrhage; HR, Heart rate; MABP, Mean arterial blood pressure; MRI, Magnetic resonance; NICU, Neonatal intensive care unit; NIRS, Near-infrared spectroscopy; PDA, Patent ductus arteriosus; RDS, Respiratory distress syndrome; $\mathrm{SpO}_{2}$ Arterial oxygen saturation measured by pulse oximetry; $\mathrm{StO}_{2}$, Regional oxygen saturation; $\mathrm{SO}_{2}$, Oxygen saturation; THI, Tissue hemoglobin index; TOI, Tissue oxygenation index; USA, United States of America.

Acknowledgment: The work was supported by a VEGA grant (No.1/0100/15) provided by Scientific Grant Agency of the Ministry of Education, science, research and sport of the Slovak Republic and the Slovak Academy of Sciences. The study was performed as a part of the Center of Excellence of Perinatology Research (CEPV II) project which is co-financed from European Union sources. The VEGA grant and the CEPV II project played no role in the design of the study and collection, analysis and interpretation of data and in writing the manuscript. Author contributions: RG: literature search and manuscript writing; RG, LCL: literature search and critical reading; all authors: manuscript revision; $\mathrm{MZ}$ : final approval.

Conflict of interest statement: The authors state that there are no conflict of interest regarding the publication of this article.

\section{REFERENCES}

1. Schneider A, Minnich B, Hofstätter E, Weisser Ch, HattingerJürgenssen $E$, Wald $M$. Comparison of four near-infrared spectroscopy devices shows that they are only suitable for 
monitoring cerebral oxygenation trends in preterm infants. Acta Pediatr 2014;103(9):934-8.

2. Kenosi M, Naulaers G, Ryan CA, Dempsey EM. Current research suggests the future looks brighter for cerebral oxygenation monitoring in preterm infants. Acta Pediatr 2015;104(3):225-31.

3. Chock VY, Davis AS. Bedside cerebral monitoring to predict neurodevelopmental outcomes. NeoReviews 2009;10(3):121-9.

4. Hitz SR. Near-infrared Spectroscopy: neonatal and perinatal applications. NeoReviews 2001;2(1):22-8.

5. Jobsis FF. Noninvasive, infrared monitoring of cerebral myocardial oxygen sufficiency and circulatory parameters. Science 1977;198(4323):1264-7.

6. Ferrari M, Giannini I, Sideri G, Zanette E. Continuous non invasive monitoring of human brain by near infrared spectroscopy. Adv Exp Med Biol 1985;191:873-82.

7. Brazy JE, Lewis DV. Changes in cerebral blood volume and cytochrome aa3 during hypertensive peaks in preterm infants. Pediatr1986;108(6):983-7.

8. Murkin JM, Arango M. Near-infrared spectroscopy as an index of brain and tissue oxygenation. Br J Anaesth 2009;103(1):3-13.

9. Wolf M, Ferrari M, Quaresima V. Progress of near-infrared spectroscopy and topography for brain and muscle clinical applications. J Biomed Opt 2007;12(6):062104.

10. Owen-Reece H, Smith M, Elwell CE, Goldstone JC. Near infrared spectroscopy. Br J Anaesth 1999;82(3):418-26.

11. Pacquini C. Near Infrared Spectroscopy: Fundamentals, Practical Aspects and Analytical Applications. J Braz Chem Soc 2003;14(2):198-219.

12. Cohn SM, Crookes BA, Proctor KG. Near-Infrared Spectroscopy in Resuscitation. J Trauma 2003;54(5):199-202.

13. Bozzetti V, Paterlini G, Meroni V, DeLorenzo P, Gazzolo D, van Be F, Visser GHA, Valsecchi MG, Tagliabue PE. Evaluation of splanchnic oximetry, Doppler flow velocimetry in the superior mesenteric artery and feeding tolerance in very low birth weight IUGR and non-IUGR infants receiving bolus versus continuous entera nutrition. BMC Pediatr 2012;12:106.

14. Kamat V. Pulse oximetry. Indian J Anaesth 2002;46(4):261-8.

15. Naulaers G, Meyns B, Miserez M, Leunens V, Van Huffel S, Casaer $\mathrm{P}$, Weindling $\mathrm{M}$, Devlieger $\mathrm{H}$. Use of tissue oxygenation index and fractional tissue oxygen extraction as non-invasive parameters for cerebral oxygenation. A validation study in piglets. Neonatology 2007;92:120-6.

16. Nicklin SE, Hassan IAA, Wickramasinghe YA, Spencer SA. The light still shines, but not that brightly? The current status of perinatal near infrared spectroscopy. Arch Dis Child Fetal Neonatal Ed 2003;88:263-8.

17. Booth EA, Dukatz Ch, Ausman J, Wider M. Cerebral and somatic venous oximetry in adults and infants. Surg Neurol Intt2010;1:75

18. Wolf M, Naulaers G, van Bel F, Kleiser S, Greisen G. A review of near infrared spectroscopy for term and preterm newborns. J Near Infrared Spectrosc 2012;20(1):43-55.

19. Liu H, Chance B, Hielscher AH, Jacques SL, Tittel FK. Influence of blood vessels on the measurement of hemoglobin oxygenation as determined by time-resolved reflectance spectroscopy. Med Phys 1995;22(8):1209-17.

20. Blasi A, Lloyd-Fox S, Johnson HH, Elwell C. Test-retest reliability of functional near infrared spectroscopy in infants. Neurophotonics 2014;1(2):025005

21. Baik N, Urlesberger B, Schwaberger B, Schmölzer GM, Mileder L, Avian A, Pichler G. Reference Ranges for Cerebral Tissue Oxygen saturation Index in Term Neonates during Immediate Neonatal Transition after Birth. Neonatology 2015;108(4):283-6.

22. Hou $X$, Ding $H$, Teng $Y$, Zhou $C$, Tang $X$, Li S, Ding H. Research on the relationship between brain anoxia at different regional oxygen saturations and brain damage using near-infrared spectroscopy. Physiol Meas 2007;28(10):1251-65

23. Kurth CD, McCann JC, Wu J, Miles L, Loepke AW. Cerebral oxygen saturation-time threshold for hypoxic-ischemic injury in piglets. Anesth Analg 2009;108(4):1268-77.

24. Lemmers PMA, van Bel F. Left-to-right differences of regional cerebral oxygen saturation measurements by near-infrared spectroscopy in neonates. Pediatr Res 2009;65(2):226-30.

25. van Vonderen JJ, Roest AA, Siew ML, Walther FJ, Hooper SB, te Pas AB. Measuring physiological changes during the transition to life after birth. Neonatology 2014;105(3):230-42.

26. Urlesberger B, Grossauer K, Pocivalnik M, Avian A, Muller W, Pichler $\mathrm{G}$. Regional oxygen saturation of the brain and peripheral tissue during birth transition of term infants. J Pediatr 2010;157(5):740-4.

27. Karen T, Wolf M, Rahel N, Haensse D, Bucher HU, Schulz G, Fauchère JC. Changes in cerebral oxygenation during early postnatal adaptation in newborns delivered by vacuum extraction measured by near-infrared spectroscopy. BMC Pediatr 2014;14(1):1.

28. Kratky E, Pichler G, Rehak T, Avian A, Pocivalnik M, Müller W, Urlesberger B. Regional cerebral oxygen saturation in newborn infants in the first 15 min of life after vaginal delivery. Physiol Meas 2012;33(1):95-102.

29. Pichler G, Binder C, Avian A, Beckenbach E, Schmölzer GM Urlesberger B. Reference ranges for regional cerebral tissue oxygen saturation and fractional oxygen extraction in neonates during immediate transition after birth. J Pediatr 2013;163(6):1558-63.

30. Pellicer A, Greisen G, Benders M, Claris O, Dempsey E, Fumagally M, Gluud C, Hagmann C, Hellström-Westas L, Hyttel-Sorensen S, Lemmers P, Naulaers G, Pichler G, Roll C, van Bel F, van Oeveren W, Skoog M, Wolf M, Austin T. The SafeBoosc phase II randomized clinical trial: a treatment guideline for targeted near-infraredderived cerebral tissue oxygenation versus standard treatment in extremely preterm infants. Neonatology 2013;104(3):171-8.

31. Roche-Labarbe N, Fenoglio A, Aggarwal A, Dehaes M, Carp SA, Franceschini MA, Grant PE. Near-infrared spectroscopy assessment of cerebral oxygen metabolism in the developing premature brain. J Cereb Blood Flow Metab 2012;32(3):481-8.

32. Tina LG, Frigiola $A$, Abella $R$, Artale $B$, Puleo $G, D^{\prime}$ Angelo $S$, Musmarra C, Tagliabue P, Li Volti G, Florio P, Gazzolo D. Near infrared spectroscopy in healthy preterm and term newborns: correlation with gestational age and standard monitoring parameters. Curr Neurovasc Res 2009;6(3):148-54.

33. Urlesberger B, Kratky E, Rehak T, Pocivalnik M, Avian A, Czihak J, Müller W, Pichler G. Regional oxygen saturation of the brain during birth transition of term infants: comparison between elective cesarean and vaginal deliveries. J Pediatr 2011;159(3):404-8.

34. Wijbenga RG, Lemmers PM, van Bel F. Cerebral oxygenation during the first days of life in preterm and term neonates: differences between different brain regions. Pediatr Res 2011;70(4):389-94.

35. van Bel F, Lemmers P, Naulaers G. Monitoring neonatal cerebral oxygen saturation in clinical practice: value and pitfalls. Neonatology 2008;94(4):237-44.

36. Sood BG, McLaughlin K, Cortez J. Near-infrared spectroscopy: Applications in neonates. Semin Fetal Neonatal Med 2015;20(3):164-72

37. McNeill S, Gatenby JC, McElroy S, Engelhardt B. Normal cerebral, renal and abdominal regional oxygen saturations using near-infrared spectroscopy in preterm infants. J Perinatol 2011;31(1):517.

38. Nanavati T, Seemaladinne N, Regier M, Yossuck P, Pergami P. Can we predict functional outcome in neonates with hypoxic ischemic encephalopathy by the combination of Neuroimaging and Electroencephalography? Pediatr Neonatol 2015;56(5):307-16.

39. Dehaes M, Aggarwal A, Lin PY, Fortuno RC, Fenoglio A, RocheLabarbe N, Soul JS, Franceschini MA, Grant EP. Cerebral oxygen metabolism in neonatal hypoxic ischemic encephalopathy during and after therapeutic hypothermia. J Cereb Blood Flow Metab 2014;34(1):87-94.

40. Plomgaard AM, van Oeveren $W$, Petersen $T H$, Alderliesten $T$, Austin T, van Bel F, Benders M, Claris O, Dempsey E, Franz A, Fumagalli M, Gluud Ch, Hagmann C, Hyttel-Sorensen S, Lemmers P, Pellicer A, Pichler G, Winkel P, Greisen G. The SafeBoosc II randomized trial: treatment guided by near-infrared spectroscopy 
reduces cerebral hypoxia without changing early biomarkers of brain injury. Pediatr Res 2016;79(4):528-35.

41. Nizarali Z, Marques T, Costa C, Barroso R, Cunha M. Ductus Arteriosus: Perinatal risk factors. J Neonatal Biol 2012;1(3):109.

42. Lemmers PMA, Toet MC, van Bel F. Impact of patent ductus arteriosus and subsequent therapy with indomethacin on cerebral oxygenation in preterm infants. Pediatrics 2008;121(1):142-7.

43. Underwood MA, Milstein JM, Sherman MP. Near-infrared spectroscopy as a screening tool for patent ductus arteriosus in extremely low birth weight infants. Neonatology 2007;91(2):134-9.

44. Vanderhaegen J, De Smet D, Meyns B, van de Velde M, van Huffel $S$, Naulaers $\mathrm{G}$. Surgical closure of the patent ductus arteriosus and its effect on the cerebral tissue oxygenation. Acta Pediatrica 2008;97(12):1640-4.

45. Lemmers PMA, Molenschot MC, Evens J, Toet MC, van Bel F. Is cerebral oxygen supply compromised in preterm infants undergoing surgical closure for patent ductus arteriosus? Arch Dis in Child Fetal Neonatal Ed 2010;95(6):429-34.
46. Lemmers PMA, Toet $M$, van Schelven LJ, van Bel F. Cerebral oxygenation and cerebral oxygen extraction in the preterm infant: the impact of respiratory distress syndrome. Exp Brain Res 2006;173(3):458-67.

47. Sorensen LC, Maroun LL, Borch K, Lou HC, Greisen G. Neonatal cerebral oxygenation is not linked to foetal vasculitis and predicts intraventricular haemorrhage in preterm infants. Acta Paediatr 2008;97(11):1529-34.

48. Zhang Y, Chan CS, Tracy MB, Lee QY, Hinder M, Savkin AV, Lovell $\mathrm{NH}$. Cerebral near-infrared spectroscopy analysis in preterm infants with intraventricular hemorrhage. Conf Proc IEEE Eng Med Biol Soc 2011;1937-40.

49. Alderliesten T, Lemmers PM, Smarius JJ, van de Vosse RE, Baerts W, van Bel F. Cerebral oxygenation, extraction, and autoregulation in very preterm infants who develop peri-intraventricular hemorrhage. J Pediatr 2013;162(4):698-704. 